\title{
Decoding Oxidative Stress and Antioxidative Parameters in Patients with Spinal Cord Injury
}

\section{Ghizal Fatima', Jan Fedacko², Ammar Mehdi Raza ${ }^{3}$, Najah R. Hadi ${ }^{4}$}

\author{
${ }^{1}$ Era's Lucknow Medical College and Hospital, Lucknow, India \\ ${ }^{2}$ Department of Cardiology, Pavol Jozef Safarik University, Kosice, Slovakia \\ ${ }^{3}$ Department of Career Institute of Medical and Dental Sciences, Lucknow, India \\ ${ }^{4}$ Department of Pharmacology and Therapeutics, College of Medicine, Kufa University, Iraq
}

Corresponding Author: Dr. Ghizal Fatima, Era's Lucknow Medical College and Hospital, Lucknow, India; Ghizalfatima8@,gmail.com; info@elmcindia.org

\section{Introduction:}

In this study we evaluated the connivance of oxidative and antioxidative parameters in the pathogenesis of spinal cord injury (SCI). Although the etiology and pathogenesis of SCI remain to be fully understood, it has been suggested that reactive oxygen species (ROS) and oxidative stress may play a significant role in the pathophysiology of SCI. Furthermore, there is little information available in scientific literature about oxidative and antioxidative parameters in SCI patients.

\section{Methods:}

Oxidative stress was determined by measuring the levels of Lipid Peroxides (LPO) and Protein carbonyl in plasma and antioxidative parameters like Glutathione Reductase (GR), catalase and Glutathione peroxidase (GPx) in lysate in 40 SCI patients and 40 healthy subjects without SCI. However, pain was measured by McGill pain questionnaire.

\section{Results:}

Concentrations of catalase $(\mathrm{p}<0.01)$, GR $(\mathrm{p}<0.01)$ and GPx $(\mathrm{p}<0.01)$ were significantly lower in patients with SCI than in controls, and levels of oxidative stress parameters, LPO $(\mathrm{p}<0.01)$, Protein carbonyl $(\mathrm{p}<0.01)$ were significantly higher in patients than in controls. A significant positive correlation was found between LPO and pain score among SCI patients group. Furthermore, a significant positive correlation was also found between Protein carbonyl and pain score among SCI patients group than in control group.

\section{Conclusion:}

The present results indicate that SCI patients are exposed to oxidative stress and this escalated oxidative stress may play a role in the etiopathogenesis of the disease. Moreover, our results also show that increased oxidative stress parameters are more strongly amalgamated with pain in SCI patients.

\section{Key words:}


Spinal Cord Injury, Oxidative stress, Antioxidants, Pain

\section{Introduction:}

Spinal cord injury (SCI) is one of the most severe traumatic injuries that results in dysfunction of limbs and trunk, below the damaged section causing myelopathy or damage to nerve roots or myelinated fiber tracts that carry signals to and from the brain. SCI leads to postoperative complications, including spinal cord edema and cell death in the injured areas, and even permanent disability and eventually death (1). Due to the recent progress in several advanced techniques, including tissue transplantation, immunotherapy and other advanced reconstruction methods, the treatment of SCI has not been yet substantially developed. Signs (observed by a clinician) and symptoms (experienced by a patient) vary depending on where the spine is injured and the extent of the injury. Furthermore, SCI prevalence is not known in India and in many other countries. According to known data, about 450,000 people in the United States live with SCI (one in 670), and there are about 11,000 new SCI every year (one in 30,000). A section of skin that is innervated through a specific part of the spine is called as dermatome, and injury to that part of the spine can cause pain, numbness, or a loss of sensation in the related areas. Paraesthesia, a tingling or burning sensation in affected areas of the skin, is another symptom of SCI.

However, the severe damage induced by SCI significantly impairs the quality of life of SCI patients. SCI includes acute and secondary injuries. The acute SCI could result in a bruise, a partial tear, or a complete tear in the spinal cord (2). The primary SCI could degrade key cytoskeletal and activate functional proteins, leading to delayed death of local and adjacent neurons and glial cells (3). Primary injury is considered to be irreversible mechanical damage (4). In contrast, the secondary injury is reversible which could be altered by potential interventions (5). Secondary lesions in severe SCI are mainly due to the inflammatory cascade activation and overproduction of free radicals (6). A complex cascade of pathophysiologic events related to free radicals, and altered blood flow accounts for clinical deterioration and poor overall outcome $(7,8)$. Therefore, large amount of studies have focused on the understanding of the mechanism of SCI-related secondary injury and developing more efficient therapeutic options that can ameliorate and even reverse secondary damage of SCI, as inflammatory responses due to oxidative stress are the crucial process of secondary injury. 
ROS are produced in the lipophilic environment of mitochondrial membranes and that antioxidants deficiency may be involved in oxidative stress in SCI $(9,10)$. It is well known that hypoxia may result in both ROS production and decrease antioxidant vitamin levels in different inflammatory diseases $(11,12)$

Significant association has been observed between protective effect of antioxidants in spinal cord injury patients $(13,14)$. However, there is little information about oxidative stress in SCI patients. Moreover, there are several disorders that are associated with oxidative stress manifested by protein oxidation, LP and other markers. Although the etiology and pathogenesis of spinal cord injury remain to be fully understood, it has been suggested that reactive oxygen species and oxidative stress have a significant role in the pathophysiology of spinal cord injury. Researchers have shown evidence that oxidative stress might play a central role in the pathophysiology of SCI. Furthermore, attempts have not been made on establishing the role of oxidative, antioxidative parameters in SCI patients which is crucial for understanding the significance of inflammation in the development of SCI. Therefore, the present study aim to investigate the oxidative parameters and antioxidative parameters contents in patients suffering from SCI.

\section{Materials and Methods:}

The study group comprised of $40 \mathrm{SCI}$ patients and 40 age matched healthy controls without SCI, who were non-alcoholic, non-smokers, non-diabetic without any kind of cardiac, respiratory and endocrinal disease, 1 weeks post injury participants were recruited. All the patients were in their acute injury phases. Patients of SCI were recruited from the spinal unit in the Department of Physical Medicine and Rehabilitation (PMR), Consultant Incharge recruited the patients, at the King George's Medical University, Lucknow, India. Besides the neurological damage to their spinal cords, each subject was physically and mentally healthy, as determined by history and physical examination, psychological questionnaires and interview with a psychologist, electrocardiogram, blood and urine chemistries, and chest radiographs. In no case was brain damage or extended loss of consciousness associated with the SCI was found.

All subjects were drug free at the time of study, including prescription, nonprescription, and over the counter medications, as well as caffeine, nicotine, and alcohol, as confirmed by urine toxicology on the first day of the inpatient study. During the week before the study, subjects 
maintained a regular sleep/wake schedule, as confirmed by patients self-report. These SCI subjects were otherwise healthy as established by medical history and physical and psychological examination. Furthermore, all subjects were male, control subjects were recruited from relatives of the patients and other normal persons of Lucknow.

\section{Institutional Ethics Committee}

Institutional Ethics Committee of King Georges Medical University approved the study, in Nov. $2016(\mathrm{kgmu} / 16 / 12 / 0129)$. Before enrolling in the study, written informed consent was obtained from both the subject groups using documents approved by the Institutional Ethics Committee and all applicable institutional and governmental regulations concerning the ethical use of human volunteers were followed during this study. All data was coded to remove any identifiable information.

Four $\mathrm{ml}$ of intravenous blood was collected from both the group of patients and controls. All the subjects completed a structured questionnaire, which assessed the biographical information, medical, personal and family history. Pain was assessed by McGill pain questionnaire (25).

\section{Oxidative and Antioxidative parameters}

Oxidative parameters were determined by measuring the levels of Lipid Peroxides (LPO) by the method given by Ohkawa et al 1979: and Protein carbonyl group was measured by the method given by Levine and Williams, 1994 in plasma whereas antioxidative parameters were determined by measuring the levels of Catalase measured by the method illustrated by Aebi 1974, whereas GPx was measured by the method of Pagila and Valentine; 1967 and GR was measured by the method of Hazelton and Lang; 1995 in lysate in 40 patients and in equal number of healthy controls.

\section{Statistical analysis}

Statistical analysis was done using SPSS statistical software (16.0 versions). Quantitative variables of SCI patients and controls were presented as the mean \pm standard deviation, and are compared by independent t-test. Pearson correlation was done to find the pattern of associations between the groups. A value of $\mathrm{p}<0.05$ was considered statistically significant and $\mathrm{p}<0.01$ is considered highly significant.

\section{Results:}




\section{Oxidative Parameters:}

A significantly higher level of LPO ( $\mathrm{p}<0.01)$, and protein carbonyl group $(\mathrm{p}<0.01)$, (Table 1) were found among patients group than in control group. Pain score was significantly higher in patients than in control group (data not shown). A significant positive correlation was found between LPO and pain score among SCI patients group. Furthermore, a significant positive correlation was also found between Protein carbonyl group and pain score among SCI patients group than in control group (Table-3).

Table1: Oxidative stress parameters among study and control group

\begin{tabular}{|c|c|c|c|}
\hline $\begin{array}{c}\text { Oxidative stress } \\
\text { parameters }\end{array}$ & $\begin{array}{c}\text { SCI patients } n=40 \\
\text { Mean } \pm \text { SD }\end{array}$ & $\begin{array}{c}\text { Control } n=40 \\
\text { Mean } \pm \text { SD }\end{array}$ & p-value \\
\hline $\begin{array}{c}\text { Lipid Peroxides } \\
\text { (LPO) }\end{array}$ & $4.8 \pm 0.8$ & $2.9 \pm 0.4$ & $<0.01$ \\
\hline Protein carbonyl & $2.8 \pm 0.7$ & $1.7 \pm 0.4$ & $<0.01$ \\
\hline
\end{tabular}

Antioxidative parameters:

A significantly lower levels of catalase $(p<0.01)$, GR $(p<0.01)$ and GPx $(p<0.01)$ (Table 2) were found among patients group than in control group.

Table 2: Antioxidative parameters among study and control group

\begin{tabular}{|c|c|c|c|}
\hline $\begin{array}{c}\text { Antioxidative } \\
\text { parameters }\end{array}$ & $\begin{array}{c}\text { SCI patients } \boldsymbol{n}=\mathbf{4 0} \\
\text { Mean } \pm \text { SD }\end{array}$ & Mean \pm SD & p-value \\
\hline Catalase & $39.0 \pm 10.4$ & $51.5 \pm 11.9$ & \\
\hline Glutathione \\
peroxidase (GPx)
\end{tabular}


Table 3: Pearson correlation analysis in between Oxidative stress parameters, Antioxidative parameters and age and Pain.

\begin{tabular}{|c|c|c|c|}
\hline \multirow{2}{*}{$\begin{array}{c}\text { Oxidative and } \\
\text { Antioxidative } \\
\text { Parameters }\end{array}$} & \multirow[b]{2}{*}{ Groups } & Age & Pain \\
\hline & & $\mathbf{r}$ & $\mathbf{r}$ \\
\hline \multirow{2}{*}{ LPO } & SCI patients & -0.033 & $-.393^{*}$ \\
\hline & Controls & 0.021 & -.161 \\
\hline \multirow{2}{*}{ Protein Carbonyl } & SCI patients & -0.042 & $-.473^{*}$ \\
\hline & Controls & 0.034 & .171 \\
\hline \multirow{2}{*}{ Catalase } & SCI patients & -0.161 & -0.199 \\
\hline & Controls & 0.193 & 0.107 \\
\hline \multirow{2}{*}{ GPx } & SCI patients & 0.051 & -0.185 \\
\hline & Controls & 0.075 & -.124 \\
\hline \multirow{2}{*}{ GR } & SCI patients & 0.072 & 0.178 \\
\hline & Controls & 0.091 & -.101 \\
\hline
\end{tabular}

$\mathrm{r}=$ Simple regression, ${ }^{*} \mathrm{p}<0.05$ (significant)

\section{Discussion:}

The present results indicate that SCI patients were under oxidative stress demonstrated with increased levels of LPO and protein carbonyl whereas decrease levels of catalase, GR and GPx among patients group than in control group. Our findings are in concurrence with report of Vaishnav et al (15) who reported significantly increased lipid peroxide in SCI patients compared to controls. Furthermore, another study reported by Liao et al. (16) showed that lipid peroxide levels were higher and superoxide dismutase levels were lower in patients with traumatic injury to spinal cord, and an imbalance existed in oxidant and antioxidant levels in SCI patients. The cause of the imbalance between oxidant and antioxidant levels in SCI is still unknown. 
Moreover, large numbers of studies have shown high levels of oxidative stress markers, such as LPO levels, in SCI patients, suggesting that this process may have a role in the pathophysiology of this disease $(17,18)$. Additionally, we have also shown the relation between oxidative stress and SCI. Efforts to explain the pathogenesis of SCI and other chronic pain conditions by oxidative stress mechanisms are not new. Visavadiya et al. (19) results indicated that by $24 \mathrm{~h}$ post-injury, ROS-induced protein oxidation is more prominent compared to lipid oxidation, indicating a critical temporal distinction in secondary pathophysiology that is critical in designing therapeutic approaches to mitigate consequences of oxidative stress in SCI; Xiong et al. (20) reported Peroxynitrite (PON, ONOO(-)), formed by nitric oxide synthase-generated nitric oxide radical ( $\mathrm{NO}$ ) and superoxide radical $(\mathrm{O}(2)(-))$, is a crucial marker in posttraumatic oxidative damage and also if any improved antioxidant neuroprotective treatment is given to these patients it will improve their post-traumatic oxidative damage in the injured spinal cord patients.

Another study by Bastani et al (21) pointed out that the levels of antioxidants were significantly lower in SCI patients, while the marker of oxidative stress was higher in the SCI subjects compared with the controls. This observation demonstrates that SCI patients experience increased oxidative stress and reduced antioxidant defense the first year after injury. Signs of oxidative stress in SCI include high levels of oxidative damage to DNA in patients with SCI. Reduced oxidative metabolism and mitochondrial abnormalities in SCI also support a mitochondrial defect as a contributor (22).

ROS are formed in mitochondria when energy metabolism is compromised, and are inactivated by the ROS scavengers, superoxide dismutase (SOD), catalase, and glutathione. ROS can impair the function of several cellular components including proteins, nucleic acids, and lipids. Moreover, since mitochondria supply energy to the cell thorough oxidative 
phosphorylation, the lower level of ATP that results from a low mitochondrial activity may explain the low exercise capacity and fatigue reported in patients with SCI (23). Free radicals and other damaging reactive oxygen species are produced in oxidative metabolic and physiological processes; their activity is also thought to increase in patients with SCI. Although the etiology of SCI is unknown, symptoms like pain may be associated with an imbalance between oxidant and antioxidant status.

It is known that LPO, as a consequence of oxidative stress, indirectly reflects intracellular ROS generation, and ROS are known to be implicated in the etiology of pain, by inducing peripheral and central hyperalgesia (24). Therefore, for this reason we thought that oxidative stress might affect the severity of SCI by increasing pain. And we investigated this issue and found a significant positive correlation between LPO and in severity of pain among patients group. Furthermore, a significant positive correlation was also found between Protein carbonyl group and pain in SCI patients group than in control group.

\section{Conclusion:}

In conclusion our study suggests an oxidant and antioxidant imbalance in SCI patients. The increase in oxidant levels and decrease in antioxidant levels show that SCI is related to free radical-mediated disorders. Accordingly, SCI patients are exposed to oxidative stress and this increased oxidative stress may play a role in the etiopathogenesis of SCI. Moreover, our results also show that increased oxidative stress parameters are more strongly associated with severity of SCI. In light of previous studies, ours study supports the hypothesis that SCI is strongly linked to increased oxidative stress. Therefore, patients with SCI are oxidatively stressed according to change of LPO and protein carbonyl. These results are consistent with the underlying hypothesis that there is an imbalance between ROS production and the antioxidant defense system in SCI patients. 
Funding: No funding was received

Declaration of Interest: The authors report no declarations of interest.

\section{References:}

1-Giuliano F, Hultling C, El MW, Smith MD, Osterloh IH, Orr M, Maytom M. Randomized trial of sildenafil for the treatment of erectile dysfunction in spinal cord injury. Sildenafil Study Group. Ann Neurol.1999;1: 15-21.

2-Alkabie S, Boileau AJ.The role of therapeutic hypothermia after traumatic spinal cord injury-a systematic review. World Neurosurg.2015; 86: 432-449.

3-Henke D, Gorgas D, Doherr MG, Howard J, Forterre F, Vandevelde M. Longitudinal extension of myelomalacia by intramedullary and subdural haemorrhage in a canine model of spinal cord injury. Spine J. 2016;16:82-90

4-Evaniew N, Belley-Cote EP, Fallah N, Noonan V, Rivers CS, Dvorak MF. Methylprednisolone for the treatment of patients with acute spinal cord injuries: A systematic review and metaanalysis. J Neurotrauma. 2016;33:468-81.

5-Chen MH, Liu YH, Xu H, Xu DW, Wang CN, Wang Y, Duan CW, Zhou Y, Kan P, Shen AG, Wang YH. Lentiviral vector-mediated p27 expression facilitates recovery after spinal cord injury. Mol Neurobiol. 2016 Nov;53:6043-6056

6-Szabo TA, Warters RD, Kadry B, Stroud RE, Matthews RG, DeSantis SM, et al. The effect of general vs spinal anesthesia on the inflammatory response in orthopedic surgery. J Rom Anest Terap Int. 2012;19:13-20.

7-Crimi E, Taccone FS, Infante T, Scolletta S, Crudele V, Napoli C. Effects of intracellular acidosis on endothelial function: an overview. J Crit Care. 2012;27:108-118.

8- Weil ZM, Gaier KR, Karelina K. Injury timing alters metabolic, inflammatory and functional outcomes following repeated mild traumatic brain injury. Neurobiol Dis. 2014;70:108-116.

9- FatimaG, SharmaVP, DasSK, Mahdi AA. Oxidative stress and antioxidative parametetrs in with spinal cord injury: implications in the pathogenesis of disease. Spinal Cord. 2015 Jan;53:3-6

10-Jia Z, Zhu H, Li J, Wang X, Misra H, Li Y, Oxidative stress in spinal cord injury and antioxidant-based intervention. Spinal Cord. 2012,50:264-74.

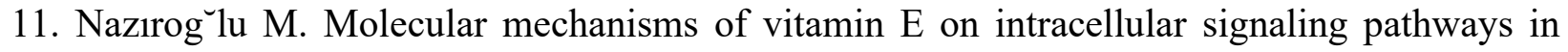
brain. In Reactive Oxygen Species and Diseases. Research Signpost Press: Kerala, India, 2007; 239-256. 
12. Halliwell B. Oxidative stress and neurodegeneration: where are we now? J Neurochem 2006; 97:1634-1658.

13- Du F, Wang X, Shang B, Fang J, Xi Y, Li A, Diao Y. Gastrodin ameliorates spinal cord injury via antioxidant and anti-inflammatory effects. Acta Biochim Pol. 2016;63:589-93.

14- Akakin D, Kiran D, Ozkan N, Erşahin M, Ozdemir-Kumral ZN, Yeğen B, Sener G. Protective effects of melatonin against spinal cord injury induced oxidative damage in rat kidney: A morphological and biochemical study. Acta Histochem. 2013 Oct;115:827-34.

15-Vaishnav RA, Singh IN, Miller DM, Hall ED. Lipid peroxidation derived reactive aldehydes directly and differentially impair spinal cord and brain mitochondrial function. J Neurotrauma 2010;27:1311-1320.

16- Liao $\mathrm{W}^{1}$, Jin XY, Wang BH, Cui XS, Wang JL, Zhang J, Zhou TJ. Impaired blood superoxide dismutase in the traumatic paraplegic patients. Acta Neurol Scand. 1992 Sep;86:32931.

17-Hall ED, Braughler JM. Role of lipid peroxidation in post-traumatic spinal cord degeneration: a review. Cent Nerv Syst Trauma. 1986 Fall;3:281-94.

18- Braughler JM, Duncan LA, Chase RL. Interaction of lipid peroxidation and calcium in the pathogenesis of neuronal injury. Cent Nerv Syst Trauma. 1985 Winter;2:269-83

19-Visavadiya NP, Patel SP, VanRooyen JL, Sullivan $\quad$ PG, Rabchevsky $\mathrm{AG}^{1}$.Cellular and subcellular oxidative stress parameters following severe spinal cord injury. Redox Biol. 2016;8:59-67.

20-Xiong Y, Rabchevsky AG, Hall ED. Role of peroxynitrite in secondary oxidative damage after spinal cord injury. J Neurochem. 2007 Feb;100:639-49.

21- Bastani NE, Kostovski E, Sakhi AK, Karlsen A, Carlsen MH, Hjeltnes N, Blomhoff R, Iversen PO. Reduced antioxidant defense and increased oxidative stress in spinal cord injured patients. Arch Phys Med Rehabil. 2012 Dec;93:2223-8

22- Ravikumar R, Flora G, Geddes JW, Hennig B, Toborek M. Nicotine attenuates oxidative stress, activation of redox-regulated transcription factors and induction of proinflammatory genes in compressive spinal cord trauma. Brain Res Mol Brain Res. 2004 May 19;124:188-98.

23- Azbill RD, Mu X, Bruce-Keller AJ, Mattson MP, Springer JE. Impaired mitochondrial function, oxidative stress and altered antioxidant enzyme activities following traumatic spinal cord injury. Brain Res. 1997 Aug 15;765:283-90. 
24- Wang ZQ, Porreca F, Cuzzocrea S, et al. A newly identified role for superoxide in inflammatory pain. J Pharmacol Exp Ther 2004;309:869-878.

25- Stein C Mendl G. The German counterpart to McGill Pain Questionnaire. Pain. 1988; 32: 251-255. 